\title{
Long-term Observation of Lateral Medullary Infarction due to Vertebral Artery Dissection Assessed with Multimodal Neuroimaging
}

\author{
Koichi Nomura ${ }^{1}$, Masahiro Mishina ${ }^{1}$, Seiji Okubo', \\ Satoshi Suda ${ }^{1}$, Ken-ichiro Katsura ${ }^{1}$ and Yasuo Katayama ${ }^{2}$ \\ ${ }^{1}$ Department of Neurological Science, Graduate School of Medicine, Nippon Medical School \\ ${ }^{2}$ Department of Neurology and Stroke Center, Tokyo General Hospital
}

\begin{abstract}
A 33-year-old man presented with a lateral medullary infarction, vertigo, and nausea. At the time of hospital admission, he had Wallenberg syndrome. Although initial magnetic resonance imaging showed no abnormalities, subsequent diffusion-weighted magnetic resonance imaging showed a high-intensity area in the right lateral medulla oblongata. The right vertebral artery was shown to be dilated on basiparallel anatomical scanning but to be stenosed on magnetic resonance angiography (MRA). Cerebral angiography 7 days after onset showed the "pearl and string sign" in the right vertebral artery. Followup MRA showed gradual improvement of the stenosis in the right vertebral artery. Multiple neuroimaging studies, such as MRA, basi-parallel anatomical scanning, 3-dimensional computed tomographic angiography, and cerebral angiography, should be performed soon after onset in suspected cases of cerebral artery dissection. In addition, serial imaging examinations increase diagnostic accuracy, and the medical history and neurological examination are important. (J Nippon Med Sch 2015; 82: 68-72)
\end{abstract}

Key words: Wallenberg syndrome, dissection, basi-parallel anatomical scanning, magnetic resonance angiography, 3-dimensional computed tomographic angiography, angiography

\section{Introduction}

Past studies reported the frequency of vertebral artery dissection was higher in Japan than Europe and the U.S. $^{1-3}$. Many patients with vertebral artery dissection often suffer ischemic stroke particularly in the lateral medulla oblongata as well as the thalamus and the cerebral or cerebellar hemisphere $\mathrm{s}^{1,3-5}$. It is widely recognized that one of the important causes of juvenile cerebral infarction is cerebral artery dissection. Patients with cerebral infarction due to the vertebral artery dissection rarely develop subarachnoid hemorrhage $e^{6,7}$, although many dissections of the vertebral arteries are thought to heal spontaneously $^{3}$. Therefore, diagnostic imaging is important to evaluate the patients' clinical condition in acute phase. However, some patients with brainstem infarction are difficult to diagnose in the acute stage using initial neuroimaging such as computed tomography (CT) and magnetic resonance imaging (MRI). In addition, vascular imagings for arterial dissection often showed various findings ${ }^{1}$. We report here a case with lateral medullary infarction due to vertebral artery dissection assessed by multimodal neuroimaging.

\section{Case Report}

A 33-year old man was referred to our hospital with vertigo and nausea. He had throbbing headache centering right occipital area since one month before, but analgesic agents were efficacious. Suddenly rotational vertigo and nausea occurred at night, when he watched television in a recumbent position. He got a rest for six hours, but vertigo and nausea were not improved. Then he was taken to our hospital. His consciousness was clear, body temperature was $36.8^{\circ} \mathrm{C}$, blood pressure was $138 / 94$ $\mathrm{mmHg}$, and heart rate was $68 / \mathrm{min}$. In the physical examination, we found no abnormality in his chest and abdomen. In the neurological examination, we observed his right Horner syndrome, gaze induced nystagmus, right curtain sign, positive Barré's sign of right upper extrem-

Correspondence to Koichi Nomura, MD, Department of Neurological Science, Graduate School of Medicine, Nippon Medical School, 1-1-5 Sendagi, Bunkyo-ku, Tokyo 113-8602, Japan

E-mail: k.nomura@nms.ac.jp

Journal Website (http://www.nms.ac.jp/jnms/) 


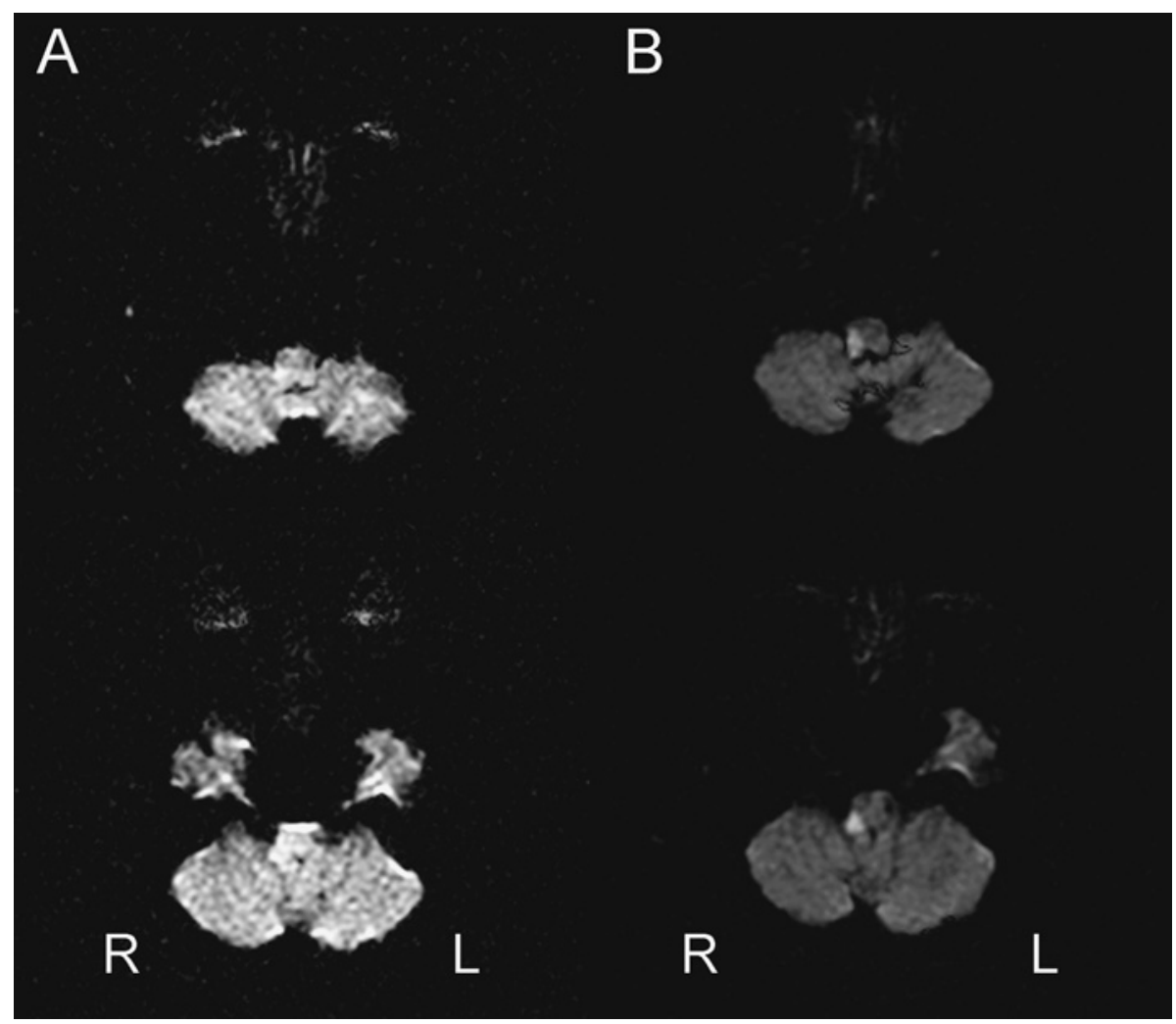

Fig. 1 Diffusion-weighted magnetic resonance images (DWIs) 7 hours (A) and 31 hours after onset (B). The initial DWIs revealed no abnormalities, but the second DWIs demonstrated a high-intensity area in the right lateral medulla oblongata.

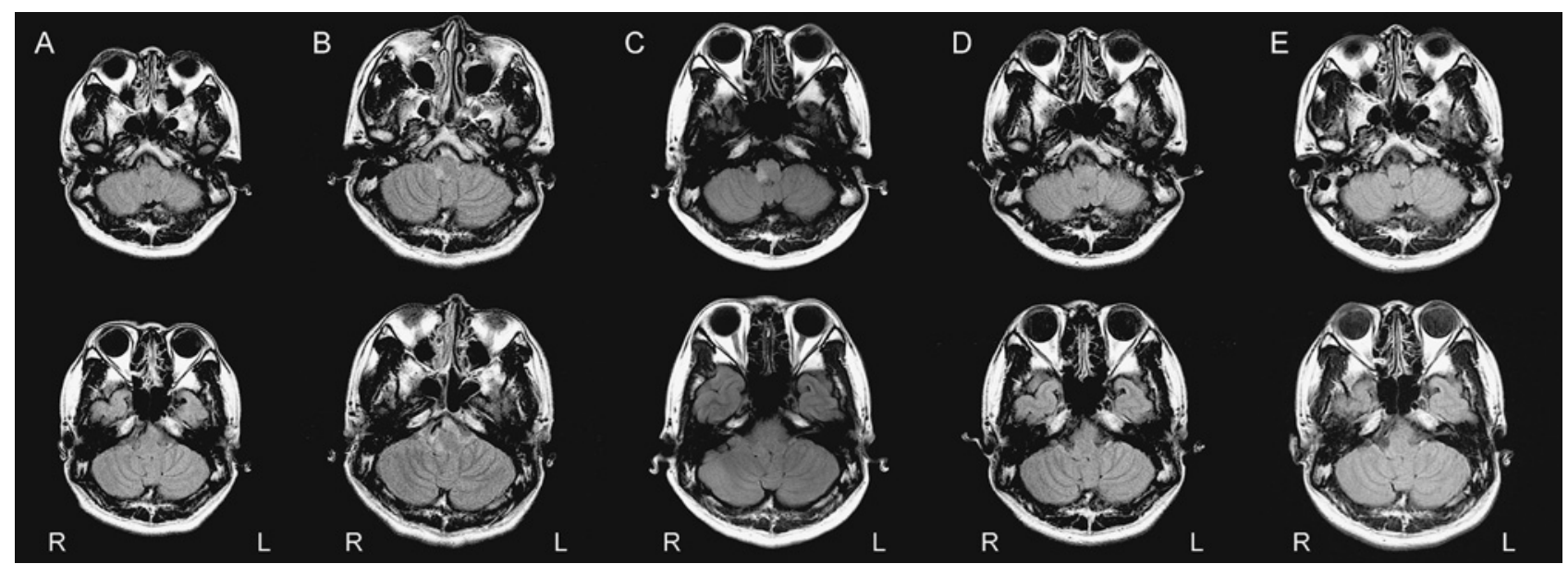

Fig. 2 Fluid attenuation inversion recovery (FLAIR) images of magnetic resonance imaging (MRI) 7 hours (A), 31 hours (B), 19 days (C), 87 days (D), and 527 days after onset (E). The initial FLAIR images showed no abnormalities; however, the second FLAIR images showed a slight high-intensity area, and the third FLAIR images showed a clear high-intensity area in the right lateral medulla oblongata. However, follow-up scans did not demonstrate the high-intensity area.

ity, cerebellar ataxia of right upper extremity, superficial sensation disorder of right face, left body trunk, and upper and lower extremities, dysarthria, dysphagia and trachyphonia. No abnormal finding was observed in blood and urinary examination, chest plain film, and electrocardiogram. We could not find abnormal findings in his head CT and MRI (Fig. 1A, 2A). However, head magnetic resonance angiography (MRA) and threedimensional computed tomographic angiography (3DCTA) showed focal stenosis at right vertebral artery (Fig. $3 \mathrm{~A}, 4)$. Based on the presence of Wallenberg syndrome and the abnormal findings of right vertebral artery, we made a diagnosis of right lateral medullary infarction due to vertebral artery dissection. MRA follow-up images 


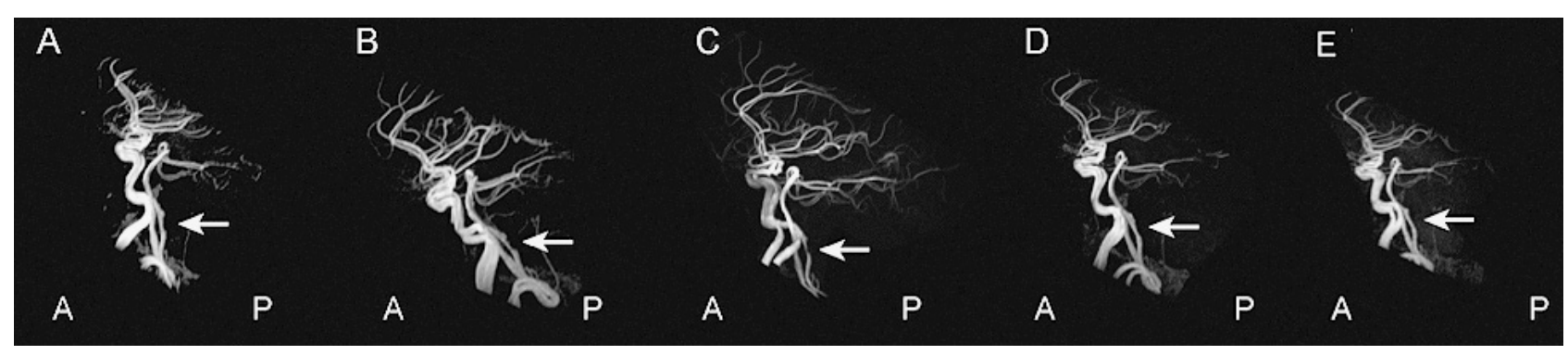

Fig. 3 Lateral magnetic resonance angiograms 7 hours (A), 31 hours (B), 19 days (C), 87 days (D), and 527 days after onset (E). Both the initial images and follow-up images showed stenosis (white arrows) and distal dilatation in the right posterior medullary segment of vertebral artery. After 87 days, the stenosis appears to have extended.

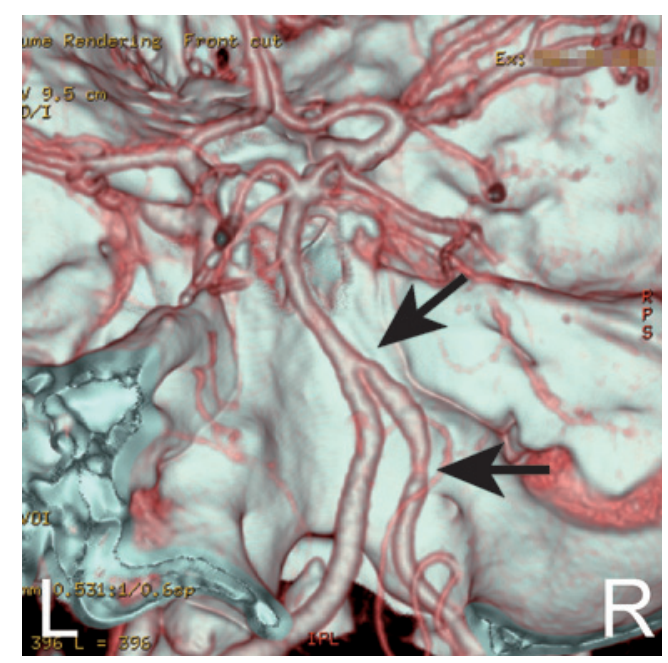

Fig. 4 Three-dimensional computed tomographic angiography 17 hours after onset. Areas of stenosis were observed in the right posterior medullary segment of the vertebral artery (lower and upper arrows).

showed that stenosis (at the white arrows) and distal dilatation in the right posterior medullary segment of vertebral artery. After 87 days, the stenosis seems to be gradually extended (Fig. 3D, 3E). We started anticoagulant therapy with continuous dosing of heparin sodium (10,000 U/day) and neuroprotective therapy with edaravone $(60 \mathrm{mg} /$ day). An MRI examination 31 hours after onset faintly showed a high-intensity area in the right lateral medulla oblongata on diffusion-weighted images (Fig. 1B) or fluid attenuation inversion recovery images (Fig. 2B). However, the high-intensity area was not seen on follow-up scans. Basi-parallel anatomical scanning (BPAS) 31 hours after onset showed dilatation of the right posterior medullary segment of vertebral artery, and BPAS after 87 days and 527 days also showed dilatation of the artery (Fig. 5A, B, C). Angiography 7 days after onset showed the "pearl and string sign" in the right posterior medullary segment of vertebral artery (Fig. 6).
The anticoagulant agent to prevent recurrence was then changed from heparin sodium to warfarin. The symptoms, including vertigo, dysarthria, and ataxia, improved. For the treatment of dysphagia, 26 days after onset the patient was transferred to a rehabilitation hospital, where he regained the ability to swallow. He returned to work, although sensory disturbance remained.

Figure 2, 3, 5 demonstrate later-follow up imaging.

\section{Discussion}

The presence of a double lumen or intimal flap on imaging is included in recent diagnostic criteria for vertebral artery dissection ${ }^{8,9}$, although angiography has been considered the gold standard for diagnosis ${ }^{1,2}$. In the present case, angiography demonstrated the "pearl and string sign" in the vertebral artery, although we could not find a double lumen or intimal flap with any imaging technique. In this case BPAS was helpful for diagnosing vertebral artery dissection. For BPAS, an MRI technique developed by Nagahara et al., only a 2-cm-thick, heavily T2-weighted coronal image, parallel to the clivus, is required $^{10}$. Imaging with BPAS can show the outer contour of the intracranial vertebrobasilar artery, whereas angiography and conventional MRA demonstrate the inner contour of the vessel. In vertebral artery dissection, the intravascular lumen (shown with conventional MRA) is narrow, and the surface of the vessel (shown with BPAS) is extended. Adding BPAS to conventional MRA sequences can improve diagnostic capability and sensitivity in suspected cases of vertebral artery dissection and is helpful for differentiating dissection from other causes of vertebral artery narrowing, such as atherosclerosis and hypoplasia ${ }^{11}$. Combined with the MRA, therefore, BPAS is a potent noninvasive examination for diagnosing vertebral artery dissection.

With regard to medical therapy for cerebral artery dissection, no clear evidence at present supports acute- 


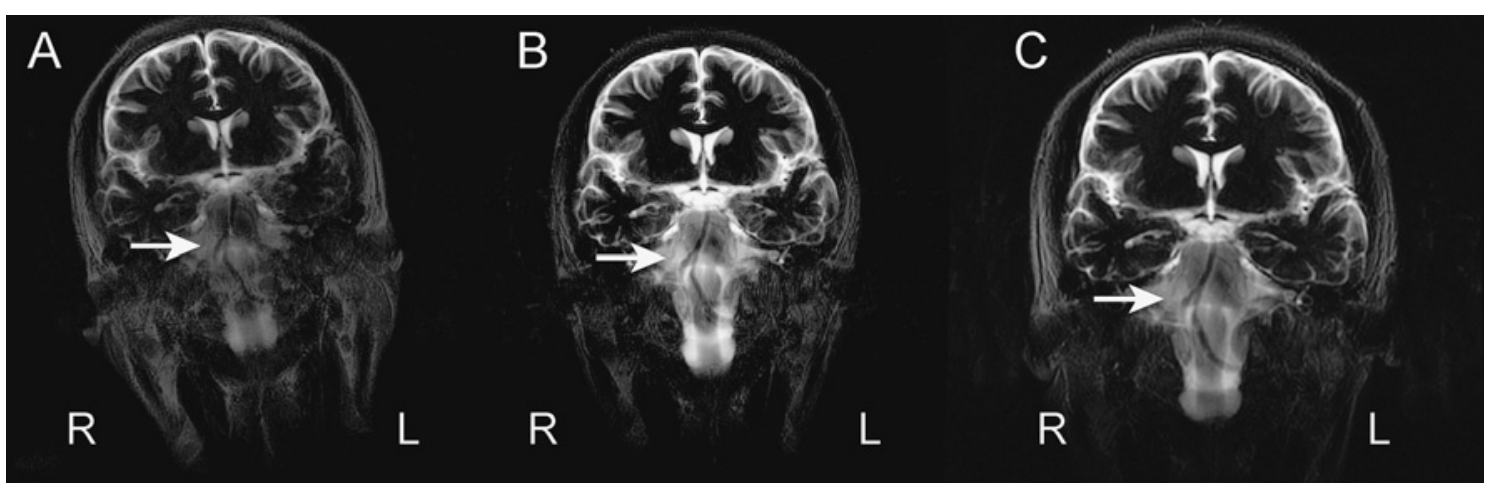

Fig. 5 Basi-parallel anatomical scanning 31 hours (A), 87 days (B), and 527 days after onset (C). Both the initial images and follow-up images showed dilatation (white arrows) in the right posterior medullary segment of the vertebral artery.

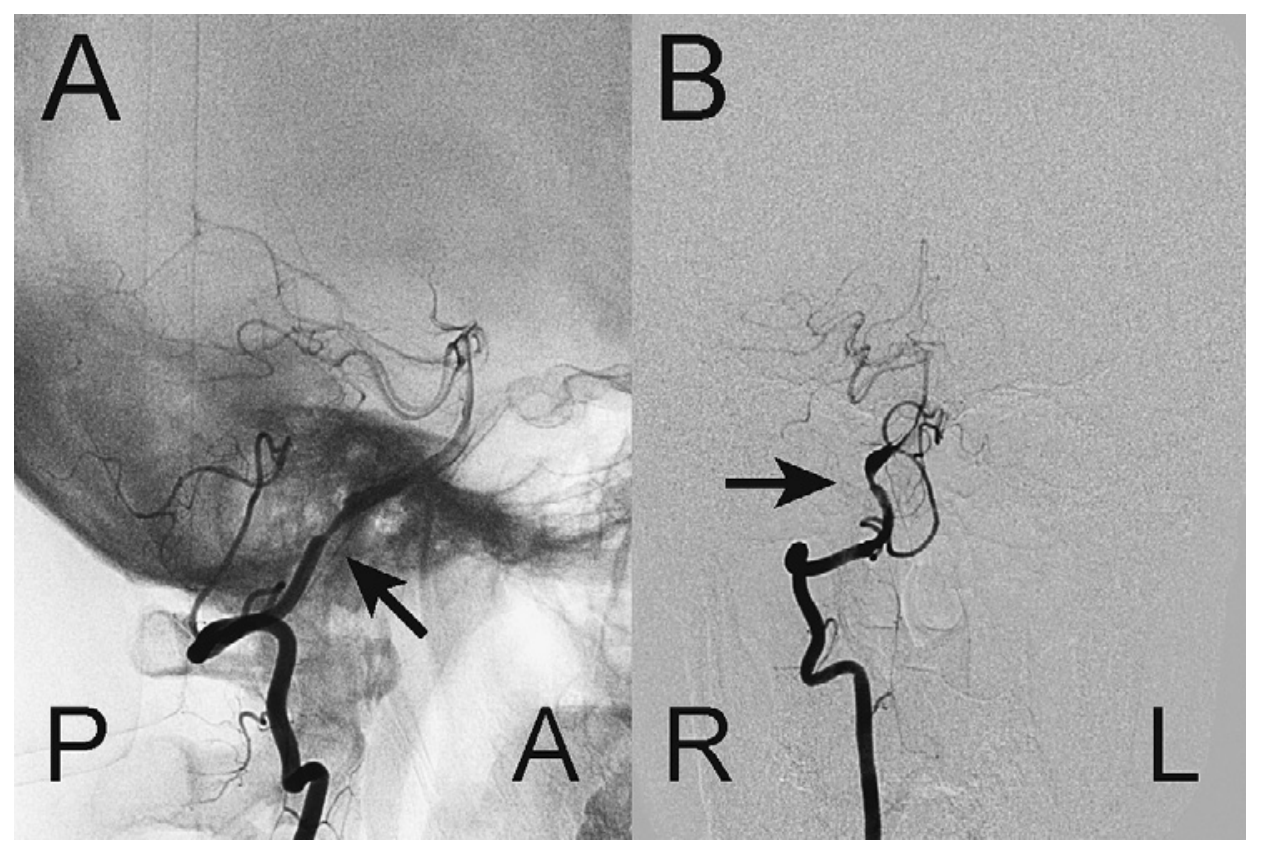

Fig. 6 Lateral view (A) and anteroposterior view (B) of right vertebral angiography 7 days after onset. Stenosis (black arrows) and distal dilatation, i.e., "pearl and string sign" is visible in the right posterior medullary segment of the vertebral artery. Note that the stenosis occurs immediately after the artery enters the skull.

phase treatment or treatment in the chronic phase for preventing recurrence. Change in the condition of the dissecting blood vessel over time is diagnostic of cerebral artery dissection. Arauz et al. have reported that the rate of complete revascularization in cases of vertebral artery dissection is $62 \% 6$ months after onset ${ }^{12}$. Lee et al. have reported that the rate of recurrence of cerebral infarction decreases 3 to 6 months after the onset of cerebral artery dissection $^{13}$. Thus, with regard to antithrombotic treatment after the acute period, imaging studies, such as MRI, should be performed and the antithrombotic regimen should be changed on the basis of the findings when appropriate. In the present case, MRA 87 days after onset showed that the stenosis had improved and suggested that the dissected blood vessel had normalized. Therefore, warfarin was discontinued. Subsequent MRA showed further improvement of the stenosis (Fig. 6).

The lateral medullary syndrome in our patient was atypical, because of the ipsilateral hemiparesis. In this case, ipsilateral motor nerves can be disturbed by the extension of the ischemia from the right medulla to the upper cervical spinal cord involving corticospinal fibers caudal to the pyramidal decussation ${ }^{14}$.

In the present case, initial diffusion-weighted MRI showed no significant abnormalities. Oppenheim et al. have reported that only $10 \%$ of initial diffusion-weighted 
images in cases of medullary infarct show evidence of infarct less than 8 hours after onset ${ }^{15}$. For the reason above, diagnosing brainstem infarction with only the findings of MRI at the initial visit is difficult. Therefore, the medical history and neurological findings are important for establishing a diagnosis. Multiple neuroimaging studies, such as MRA, BPAS, 3D-CTA, and cerebral angiography, should be performed soon after onset in suspected cases of cerebral artery dissection. In addition, serial imaging examinations increases the accuracy of diagnosis.

Conflict of Interest: The authors declare no conflict of interest.

\section{References}

1. Yamaura A, Yoshimoto T, Hashimoto N, Ono J: Nationwide Study of Nontraumatic Intracranial Arterial Dissection: Clinical Features and Outcome. Surgery for Cerebral Stroke 1988; 26: 79-86.

2. Yamaura A, Yoshimoto T, Hashimoto N, Ono J: Nationwide Study of Nontraumatic Intracranial Arterial Dissection: Treatment and Its Results. Surgery for Cerebral Stroke 1988; 26: 87-95.

3. Schievink WI: Spontaneous dissection of the carotid and vertebral arteries. The N Engl J Med 2001; 344: 898-906.

4. Nakagawa $\mathrm{K}$, Touho $\mathrm{H}$, Morisako $\mathrm{T}$, et al.: Long-term follow-up study of unruptured vertebral artery dissection: clinical outcomes and serial angiographic findings. J Neurosurg 2000; 93: 19-25.

5. Naito I, Iwai T, Sasaki T: Management of intracranial vertebral artery dissections initially presenting without subarachnoid hemorrhage. Neurosurgery 2002; 51: 930-938.

6. Takumi I, Mizunari T, Mishina M, et al.: Dissecting posterior inferior cerebellar artery aneurysm presenting with subarachnoid hemorrhage right after anticoagulant and antiplatelet therapy against ischemic event. Surg Neurol 2007; 68: 103-107.
7. Suzuki K, Mishina M, Okubo S, et al.: Anterior cerebral artery dissection presenting subarachnoid hemorrhage and cerebral infarction. J Nippon Med Sch 2012; 79: 153158.

8. Minematsu K, Matsuoka H, Kasuya J: Cervicocephalic arterial dissection in Japan: analysis of 454 patients in the spontaneous cervicocephalic arterial dissection study I (SCADS-I). Stroke 2008; 39: 566.

9. Sato S, Toyoda K, Matsuoka $\mathrm{H}$, et al.: Isolated anterior cerebral artery territory infarction: dissection as an etiological mechanism. Cerebrovasc Dis 2010; 29: 170-177.

10. Nagahata M, Abe Y, Ono S, Hosoya T, Uno S: Surface appearance of the vertebrobasilar artery revealed on basiparallel anatomic scanning (BPAS)-MR imaging: its role for brain MR examination. AJNR Am J Neuroradiol 2005; 26: $2508-2513$.

11. Fatima Z, Motosugi U, Okumura A, Ishigame $\mathrm{K}$, Araki T: Basi-parallel anatomical scanning (BPAS)-MRI can improve discrimination of vertebral artery dissection from atherosclerosis and hypoplasia. Acad Radiol 2012; 19: 1362-1367.

12. Arauz A, Marquez JM, Artigas C, Balderrama J, Orrego $\mathrm{H}$ : Recanalization of vertebral artery dissection. Stroke 2010; 41: 717-721.

13. Lee VH, Brown RD Jr, Mandrekar JN, Mokri B: Incidence and outcome of cervical artery dissection: a populationbased study. Neurology 2006; 67: 1809-1812.

14. Garcia-Garcia J, Ayo-Martin O, Segura T: Lateral medullary syndrome and ipsilateral hemiplegia (Opalski syndrome) due to left vertebral artery dissection. Arch Neurol 2009; 66: 1574-1575.

15. Oppenheim C, Stanescu R, Dormont D, et al.: Falsenegative diffusion-weighted $\mathrm{MR}$ findings in acute ischemic stroke. AJNR Am J Neuroradiol 2000; 21: 14341440.

(Received, June 26, 2014)

(Accepted, October 6, 2014) 